\title{
Measuring Shape Circularity
}

\author{
Joviša Žunić ${ }^{1, \star}$ and Kaoru Hirota ${ }^{2}$ \\ ${ }^{1}$ Computer Science, Exeter University, Harrison Building, Exeter EX4 4QF, U.K. \\ J.Zunic@ex.ac.uk \\ ${ }^{2}$ Department of Computational Intelligence and Systems Science, Graduate School of \\ Science and Engineering, Tokyo Institute of Technology, G3-49, 4259 Nagatsuta, \\ Modori-ku, Yokohama 226-8502, Japan \\ hirota@hrt.dis.titech.ac.jp
}

\begin{abstract}
In this paper we define a new circularity measure. The new measure is easy to compute and, being area based, is robust with respect to noise. It ranges over $(0,1]$ and gives the measured circularity equal to 1 if and only if the measured shape is a circle. The new measure is invariant with respect to translations, rotations and scaling.
\end{abstract}

Keywords: shape, circularity measure, moments.

\section{Introduction}

Shape descriptors are a powerful tool for shape classification tasks. Many shape descriptors are already studied in the literature and used in practice. Some of the most known ones are: elongation $([13)$, convexity $(9])$, rectangularity $([10])$, rectilinearity $([\mathbf{1 6})$, sigmoidality $([1])$, etc.

In this paper we define a new circularity measure. Several circularity measures already exist in the literature ( $\left.\begin{array}{llll}1 & 2 & 3 & 4\end{array}\right]$ ). Probably the most standard circularity measure (sometimes also called the compactness) is derived from the relation between the shape area and its perimeter. Taking into account that among all shapes with the same area, the circle has the minimal perimeter, someone can conclude that the shape circularity measure $\mathcal{C}_{s t}(S)$ can be defined as

$$
\mathcal{C}_{s t}(S)=\frac{4 \cdot \pi \cdot \text { area_of_S }}{(\text { perimeter_of_S })^{2}} .
$$

It is easy to check that the following natural requirements, for such a defined circularity measure, hold:

(a) $\mathcal{C}_{s t}(S) \in(0,1]$;

(b) $\mathcal{C}_{s t}(S)=1$ if and only if $S$ is a circle;

(c) $\mathcal{C}_{s t}(S)$ is invariant with respect to similarity transformations (e.g. translations, rotations and scaling).

\footnotetext{
* J. Žunić is also with the Mathematical institute of Serbian Academy of Sciences and Arts, Belgrade.
} 
(d) For each $\delta>0$ there is a shape $S$ such that $0<\mathcal{C}_{s} t(S)<\delta$, i.e. there are shapes whose measured circularity is arbitrary close to 0 .

Geometric moments will be used in the definition of the new circularity measure. Just to mention that geometric moments are widely used in computer vision, image analysis, or pattern recognition tasks ([7]). The $(p, q)$-moment $m_{p, q}(S)$ of a planar set $S$ is defined by the following:

$$
m_{p, q}(S)=\iint_{S} x^{p} y^{q} d x d y
$$

and has the order $p+q$. Basic shape features (e.g., size, position, orientation, elongation) are computed from the moments having the order less or equal to two. E.g., $m_{0,0}(S)$ equals the area of $S$ while $\left(\frac{m_{1,0}(S)}{m_{0,0}(S)}, \frac{m_{0,1}(S)}{m_{0,0}(S)}\right)$ is the centroid of $S$. Higher order moments are needed for computing, for example, the orientation of rotationally symmetric shapes ([14) or moment invariants ([6]).

The normalised $(p, q)$-moment $\mu_{p, q}(S)$ is defined by:

$$
\mu_{p, q}(S)=\iint_{S}\left(x-\frac{m_{1,0}(S)}{m_{0,0}(S)}\right)^{p}\left(y-\frac{m_{0,1}(S)}{m_{0,0}(S)}\right)^{q} d x d y .
$$

Of course, if the centroid of $S$ is coincident with the origin then $m_{p, q}(S)=$ $\mu_{p, q}(S)$ for all $p$ and $q$. Trivially, $m_{0,0}(S)=\mu_{0,0}(S)$ but in the rest of the paper we will use $\mu_{0,0}(S)$ rather than $m_{0,0}(S)$ for the area of $S$.

In this paper we show that the quantity $\mathcal{C}(S)=\frac{\left(\mu_{0,0}(S)\right)^{2}}{2 \pi \cdot\left(\mu_{2,0}(S)+\mu_{0,2}(S)\right)}$ can be used as a circularity measure. We show that the basic requirements (a),(b),(c), and (d) (defined above) are also satisfied by $\mathcal{C}(S)$. Several examples are given to demonstrate the behaviour of the new measure and its robustness with respect to the noise.

Let us notice that the quantity $\frac{\mu_{2,0}(S)+\mu_{0,2}(S)}{\left(\mu_{0,0}(S)\right)^{2}}$ is well known from the literature. It is one of the famous Hu's invariants ([6]) adopted to be scaling invariant. The same quantity also appears as one of geometric invariants studied in 15. It is worth mentioning that the moment invariants have been used in many classification tasks, but also some of them are used to measure shape properties. E.g. [10] uses the projective invariant $I_{1}(S)=\frac{\mu_{2,0}(S) \cdot \mu_{0,2}(S)-\left(\mu_{1,1}(S)\right)^{2}}{\left(\mu_{0,0}(S)\right)^{4}}$ (see [5]) to define an ellipcity measure, under the argument that any ellipse can be obtained by applying an affine transform to a circle. Precisely, the ellipcity measure of a given shape $S$ is obtained by comparing $I_{1}(S)$ with $\frac{1}{16 \pi^{2}}=I_{1}$ (circle). The same paper derives a triangularity measure by the same reasoning and by using $I_{1}(S)$ again.

Such defined ellipcity and triangularity measures are adopted to range over $[0,1]$ and peaking at 1 for a perfect ellipse (i.e. for a perfect triangle). The problem is that, for both measures, if the measured ellipcity (triangularity) equals 1 
it is not guarantied that the measured shape is a perfect ellipse (triangle). The measure described here does not have such a disadvantage - it equals 1 if and only if the measured shape is a circle.

The paper is organised as follows. The next section introduces the new circularity measure and proves several desirable properties of it. Section 3 gives several examples which demonstrate the behaviour of the new measure $\mathcal{C}(S)$ and illustrate particular situations where $\mathcal{C}(S)$ acts differently from the standard $\mathcal{C}_{s t}(S)$ measure. Comments and conclusion are in the last section.

\section{New Circularity Measure}

In this section we define a new circularity measure. Through the paper we assume that all appearing shapes are planar and compact - what is not a restriction in image processing tasks. We start with the quantity $\frac{\mu_{2,0}(S)+\mu_{0,2}(S)}{\left(\mu_{0,0}(S)\right)^{2}}$ and show that it reaches the minimum value of $1 /(2 \pi)$ if and only if $S$ is a circle. Exploiting this fact we will come to a new circularity measure. First, we prove the following theorem.

Theorem 1. Let a given planar compact shape $S$. Then

$$
\begin{aligned}
& \frac{\mu_{2,0}(S)+\mu_{0,2}(S)}{\left(\mu_{0,0}(S)\right)^{2}} \geq \frac{1}{2 \pi} \\
& \frac{\mu_{2,0}(S)+\mu_{0,2}(S)}{\left(\mu_{0,0}(S)\right)^{2}}=\frac{1}{2 \pi} \quad \text { if and only if } S \text { is a circle. }
\end{aligned}
$$

Proof. Let a given planar compact shape $S$ whose centroid coincides with the origin. Further, let $C$ be the circle with radius $r=\sqrt{\frac{\mu_{0,0}(S)}{\pi}}$ (i.e. the areas of $C$ and $S$ are the same) and centred at the origin. Trivially:

(i) The areas of the set differences $S \backslash C$ and $C \backslash S$ are the same (because the areas of $S$ and $C$ are the same);

(ii) The points from $C \backslash S$ are closer to the origin than the points from $S \backslash C$. Or, more formally: If $(u, v) \in S \backslash C$ and $(w, z) \in C \backslash S$ then $u^{2}+v^{2}>w^{2}+z^{2}$ (see Fig. 1).

Further, ( i) and (ii) give:

$$
\iint_{S \backslash C}\left(x^{2}+y^{2}\right) d x d y \geq \iint_{C \backslash S}\left(x^{2}+y^{2}\right) d x d y .
$$

Now, we derive

$$
\mu_{2,0}(S)+\mu_{0,2}(S)=\iint_{S}\left(x^{2}+y^{2}\right) d x d y
$$




$$
\begin{aligned}
& =\iint_{S \backslash C}\left(x^{2}+y^{2}\right) d x d y+\iint_{S \cap C}\left(x^{2}+y^{2}\right) d x d y \\
& \geq \iint_{C \backslash S}\left(x^{2}+y^{2}\right) d x d y+\iint_{S \cap C}\left(x^{2}+y^{2}\right) d x d y \\
& =\iint_{C}\left(x^{2}+y^{2}\right) d x d y \\
& =4 \cdot \int_{\phi=0}^{\pi / 2} \int_{\rho=0}^{\sqrt{\mu_{0,0}(S) / \pi}}\left((\rho \cdot \cos \phi)^{2}+(\rho \cdot \sin \phi)^{2}\right) \rho d \phi d \rho \\
& =\frac{\left(\mu_{0,0}(S)\right)^{2}}{2 \pi} .
\end{aligned}
$$

This proves (2).

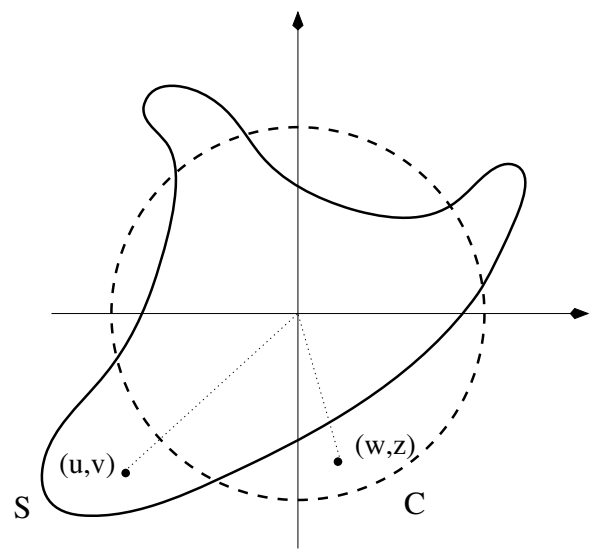

Fig. 1. The points from $C \backslash S$ are closer to the origin than the points from $S \backslash C$

To prove (3) notice that $S$ and $C$ are both compact and $S \neq C$ would imply that the inequality (4) is strict. So, $S \neq C$ implies

$$
\mu_{2,0}(S)+\mu_{0,2}(S)>\mu_{2,0}(C)+\mu_{0,2}(C)=\frac{\left(\mu_{0,0}(S)\right)^{2}}{2 \pi}
$$

for all shapes different from circles. This establishes the proof.

Thus, Theorem 1 says that $\frac{\mu_{2,0}(S)+\mu_{0,2}(S)}{\left(\mu_{0,0}(S)\right)^{2}}$ reaches its minimum if and only if $S$ is a circle. This minimum is $1 /(2 \pi)$. Based on this nice property we give the following definition for a new circularity measure.

Definition 1. The circularity measure $\mathcal{C}(S)$ of a given shape $S$ is defined as

$$
\mathcal{C}(S)=\frac{1}{2 \pi} \cdot \frac{\left(\mu_{0,0}(S)\right)^{2}}{\mu_{2,0}(S)+\mu_{0,2}(S)}
$$


The following theorem summarises desirable properties of $\mathcal{C}(S)$.

Theorem 2. Let a planar compact shape $S$. Then, the circularity measure $\mathcal{C}(S)$ satisfies the following properties:

(a) $\mathcal{C}(S) \in(0,1]$;

(b) $\mathcal{C}(S)=1 \quad \Leftrightarrow \quad S$ is a circle;

(c) $\mathcal{C}(S)$ is invariant with respect to the similarity transformations;

(d) For each $\delta>0$ there is a shape $S$ such that $0<\mathcal{C}(S)<\delta$, i.e. there are shape whose measured circularity is arbitrary close to 0.

Proof. The first two items follow directly from Theorem 1.

The third item follows from the fact that $\frac{\mu_{2,0}(S)+\mu_{0,2}(S)}{\left(\mu_{0,0}(S)\right)^{2}}$ is invariant with respect to similarity transformations. So, $\frac{1}{2 \pi} \cdot \frac{\left(\mu_{0,0}(S)\right)^{2}}{\mu_{2,0}(S)+\mu_{0,2}(S)}$ is also such an invariant.

To prove the last item let us consider a rectangle $T(t)$ whose edge lengths are 1 and $t$, where $t$ is an arbitrary positive number. It is easy to verify that

$$
\mathcal{C}(T(t))=\frac{1}{2 \pi} \cdot \frac{\left(\mu_{0,0}(T(t))\right)^{2}}{\mu_{2,0}(T(t))+\mu_{0,2}(T(t))}=\frac{6 \cdot t}{\pi \cdot\left(t^{2}+1\right)} .
$$

A trivial equality: $\lim _{t \rightarrow \infty} \mathcal{C}(T(t))=0$ establishes the proof of $(\mathbf{d})$.

Remark. Notice that the measured circularity $\mathcal{C}(T(t))=\frac{6 \cdot t}{\pi \cdot\left(t^{2}+1\right)}$ is in accordance with our perception. The highest measured circularity $\mathcal{C}(T(t))$ is for $t=1$, i.e. among all rectangles, the square has the highest measured circularity. This is as expected. Also, when $t \rightarrow \infty$ and $t \rightarrow 0$ the measured circularity tends to 0 . In those cases the rectangle degenerates into an infinitely long and constant-width strip $(t \rightarrow \infty)$ or into a line segment $(t \rightarrow 0)$, and such a measured circularity, tending to 0 , seems to be very reasonable.

\section{Experiments Illustrating $\mathcal{C}(S)$ Measure}

Examples in Fig.2 illustrate how the new circularity measure acts. Ten fish shapes are ranked with respet to their measured $\mathcal{C}(S)$ circularity (the numbers given immediately below the shapes). It could be said that the obtained ranking is in accordance with our perception.

If the same shapes are ranked with respect to the standard circularity measure $\mathcal{C}_{s t}(S)$, a different ranking $(\mathrm{b})(\mathrm{a})(\mathrm{c})(\mathrm{d})(\mathrm{e})(\mathrm{g})(\mathrm{h})(\mathrm{f})(\mathrm{i})(\mathrm{j})($ instead of $(\mathrm{a})(\mathrm{b})(\mathrm{c})(\mathrm{d})$ $(\mathrm{e})(\mathrm{f})(\mathrm{g})(\mathrm{h})(\mathrm{i})(\mathrm{j}))$ is obtained. Such a different ranking is expected. Indeed, the standard circularity measure $\mathcal{C}_{s t}(S)$ penalises deep intrusions into the shape. Such intrusions (and protrusions) lead to an essential perimeter increase and, consequently, imply a lower measured circularity. That exactly happend here. Shape in Fig.2(a), has a higher measured $\mathcal{C}(S)$ circularity than the shape in 


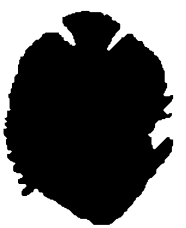

0.9579

(0.4881)

(a)

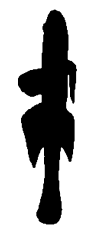

0.3810

(0.1981)

(f)

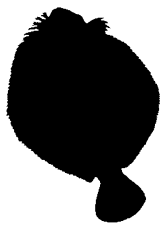

0.8755

(0.4937)

(b)

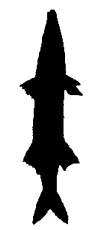

0.3361

(0.2148)

(g)

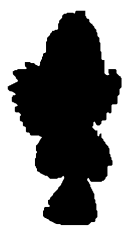

0.6765

(0.3508)

(c)

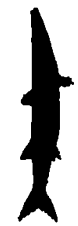

0.2776

(0.2082)

(h)

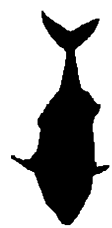

0.4506

(0.2733)

(d)

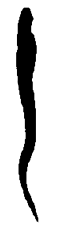

0.1390

(0.1545)

(i)

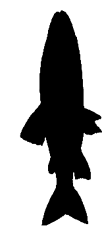

0.4385

(0.2679)

(e)

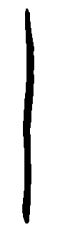

0.0729

$(0.0982)$

(j)

Fig. 2. Fish shapes are ranked with respect to their measured $\mathcal{C}(S)$ circularities. Circularities measured by $\mathcal{C}_{s t}(S)$ are in brackets.

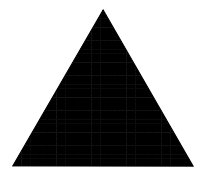

0.8265

(0.6046)

(a)

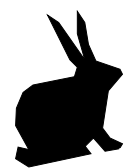

0.7459

$(0.2622)$

(f)

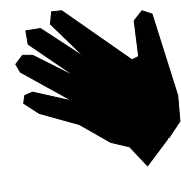

0.8174

(0.2335)

(b)

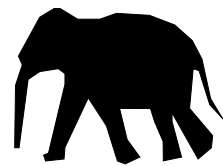

0.7387

$(0.1651)$

(g)

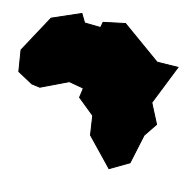

0.7851

(0.5772)

(c)

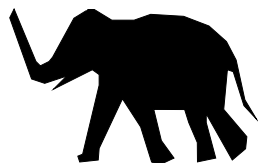

0.6967

(0.1553)

(h)

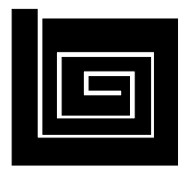

0.7789

(0.0651)

(d)

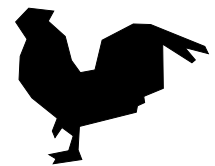

0.6600

$(0.2506)$

(i)

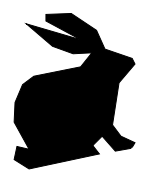

0.7777

(0.2677)

(e)

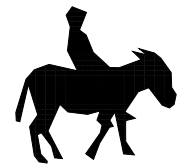

0.6142

$(0.1102)$

(j)

Fig. 3. Shapes are ranked with respect to their measured $\mathcal{C}(S)$ circularities. Circularities measure by $\mathcal{C}_{s t}(S)$ are in brackets.

Fig.2(b). On the other side, the measure $\mathcal{C}_{s t}(S)$ penalises intrusions into the shape in Fig.2(a) and assigns a higher measured circularity $\mathcal{C}_{s t}(S)$ to the shape in Fig.2(b). The same reasons lead to the 8-th position of the shape in Fig.2(f) if ranked by $\mathcal{C}_{s t}(S)$ measure. 
Several more shapes are presented in Fig.3. The same reasoning applies. The biggest difference in the measured circularities $\mathcal{C}(S)$ and $\mathcal{S}_{s t}(S)$ are in the case of shapes that have deep intrusions. Ranking $(\mathrm{a})(\mathrm{b})(\mathrm{c})(\mathrm{d})(\mathrm{e})(\mathrm{f})(\mathrm{g})(\mathrm{h})(\mathrm{i})(\mathrm{j})$ by $\mathcal{C}(S)$ is replaced by the ranking $(\mathrm{a})(\mathrm{c})(\mathrm{e})(\mathrm{f})(\mathrm{i})(\mathrm{b})(\mathrm{g})(\mathrm{h})(\mathrm{j})(\mathrm{d})$. A highly ranked shape Fig.3(d) by a use of $\mathcal{C}(S)$ is ranked the last if $\mathcal{C}_{s t}(S)$ is applied. The examples in figures Fig.2(e) and Fig.2(f) (i.e. Fig.2(g) and Fig.2(h)) illustrate how shape deformations could lead to differences in the measured circularity. In both cases the changes in the measured circularity are in accordance with our perception.

Figure 4 illustrates the robustness of the new circularity measure $\mathcal{C}(S)$. There is an original shape in Fig.4(a) and three shapes with added different level noise on it (Fig.4(b-d)). All four presented shapes have very similar measured $\mathcal{C}(S)$ circularities even that the fourth shape (in Fig.4(d)) has a very high level noise added. On the other side, the standard circularity measure $\mathcal{C}_{s t}(S)$ can cope only with a small level noise. Indeed, the shape in Fig.4(a) has an $\approx 2.3$ times higher measured circularity than the shape in Fig.4(d). Such a big difference in the measured circularity $\mathcal{C}_{s t}(S)$ is caused by a high level noise in Fig.4(d) and by the fact that $\mathcal{C}_{s t}(S)$ strongly depends on the perimeter of $S$.

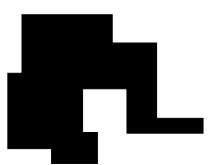

0.7470

$(0.3155)$

(a)

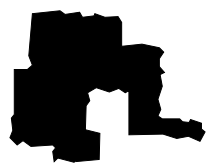

0.7520

(0.3039)

(b)

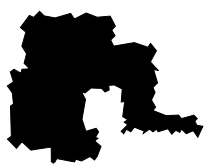

0.7565

$(0.2289)$

(c)

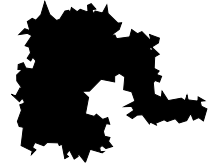

0.7412

(0.1367)

(d)

Fig. 4. Measured circularity $\mathcal{C}(S)$ of a shape with added noise. Measured $\mathcal{C}_{s t}(S)$ circularities are in brackets.

\section{Conclusion}

In this paper we introduce a new circularity measure $\mathcal{C}(S)$ defined as

$$
\mathcal{C}(S)=\frac{1}{2 \pi} \cdot \frac{\left(\mu_{0,0}(S)\right)^{2}}{\mu_{2,0}(S)+\mu_{0,2}(S)}
$$

Such a defined measure enables an easy and straightforward computation (8] ) only the moments having the order up to two are required. Being area based, the new circularity measure is robust with respect to the noise. It ranges over $(0,1]$ and gives the measured circularity equal to 1 if and only if the measured shape is a circle. The new measure is invariant with respect to translations, rotations and scaling. Several experimental results are provided to illustrate the behaviour of the new measure. The experimental results are in accordance with theoretical considerations and with our perception how a convexity measure should act. 


\section{References}

1. Di Ruberto, C., Dempster, A.: Circularity measures based on mathematical Morphology. Electronics Letters 38, 1691-1693 (2000)

2. Kim, C.E., Anderson, T.A.: Digital disks and a digital compactness measure. In: 16th Annual ACM symposium on Theory of STOC, pp. 117-124. ACM Press, New York (1984)

3. Lee, D.R., Sallee, T.: A method of measuring shape. Geographical Review 60, 555-563 (1970)

4. Proffitt, D.: The measurement of circularity and ellipticity on a digital grid. Patt. Rec. 15, 383-387 (1982)

5. Flusser, J., Suk, T.: Pattern recognition by affine moment invariants. Patt. Rec. 26, 167-174 (1993)

6. Hu., M.: Visual pattern recognition by moment invariants. IRE Trans. Inf. Theory $8,179-187(1962)$

7. Jain, R., Kasturi, R., Schunck, B.G.: Machine Vision. McGraw-Hill, New York (1995)

8. Jiang, X.Y., Bunke, H.: Simple and fast computation of moments. Patt. Rec. 24, 801-806 (1991)

9. Rahtu, E., Salo, M., Heikkila, J.: A new convexity measure based on a probabilistic interpretation of images. IEEE Trans. Patt. Anal. Mach. Intell. 28, 1501-1512 (2006)

10. Rosin, P.L.: Measuring shape: ellipticity, rectangularity, and triangularity. Machine Vision and Applications 14, 172-184 (2003)

11. Rosin, P.L.: Measuring sigmoidality. Patt. Rec. 37, 1735-1744 (2004)

12. Stojmenović, M., Nayak, A.: Shape based circularity measures of planar point sets. In: IEEE International Conference on Signal Processing and Communications, pp. 1279-1282. IEEE Press, New York (2007)

13. Stojmenović, M., Žunić, J.: Measuring Elongation from Shape Boundary. Journal Mathematical Imaging and Vision 30, 73-85 (2008)

14. Tsai, W.H., Chou, S.L.: Detection of generalized principal axes in rotationally symmetric shapes. Patt. Rec. 24, 95-104 (1991)

15. Xu, D., Li, H.: Geometric moment invariants. Patt. Rec. 41, 240-249 (2008)

16. Žunić, J., Rosin, P.L.: Rectilinearity Measurements for Polygons. IEEE Trans. on Patt. Anal. and Mach. Intell. 25, 1193-1200 (2003) 